\title{
Adipose tissue-secreted miR-27a promotes liver cancer by targeting FOXOI in obese individuals
}

This article was published in the following Dove Press journal:

OncoTargets and Therapy

7 April 2015

Number of times this article has been viewed

\author{
Baozhen Sun ${ }^{1, *}$ \\ Jing $\mathrm{Li}^{2}, *$ \\ Dan Shao ${ }^{2}$ \\ Yue $\operatorname{Pan}^{2}$ \\ Yujing Chen ${ }^{2}$ \\ Suo $\mathrm{Li}^{\prime}$ \\ Xiaoxiao Yao' \\ Hang Li ${ }^{\prime}$ \\ Weiwei Liu $^{3}$ \\ Ming Zhang ${ }^{2}$ \\ Xuewen Zhang' \\ Li Chen ${ }^{2}$ \\ 'Department of Hepatobiliary and \\ Pancreas Surgery, China-Japan Union \\ Hospital of Jilin University, Changchun, \\ People's Republic of China; \\ 2Department of Pharmacology, \\ Nanomedicine Engineering Laboratory \\ of Jilin Province, College of Basic \\ Medical Sciences, Jilin University, \\ Changchun, People's Republic of \\ China; ${ }^{3}$ School of Stomatology, Jilin \\ University, Changchun, People's \\ Republic of China \\ *These authors contributed equally \\ to this work
}

Correspondence: Xuewen Zhang Department of Hepatobiliary and Pancreas Surgery, China-Japan Union Hospital of jilin University, No 126 Xinmin Street, Chaoyang District, Changchun I30033,

People's Republic of China

Email zxw515@sohu.com

Li Chen

Department of Pharmacology, Nanomedicine Engineering Laboratory of Jilin Province, College of Basic Medical Sciences, Jilin University, No 126 of

Xiantai Street, Erdao District, Changchun

| 3002 I, People's Republic of China

Tel +86 43I 856I 9799

Fax +86 43I 856I 9799

Email chenl@jlu.edu.cn
Abstract: The current notion that obesity is a major risk factor for the development of and the mortality associated with a subset of liver cancer is well appreciated. However, detailed mechanistic insights underlying this relationship are lacking. Better understanding of the adipose tissue-secreted miRNAs that play a potential role in defining primary liver cancer development and mediating the obesity-cancer communication offers the potential for new insights into tumor growth and interventions to modulate tumor formation and progression. In this study, we clearly demonstrated that miR-27a is more highly upregulated in cancer, plasma, and adipose samples from obese liver cancer cases, and therefore reasoned that miR-27a excreted from adipose tissue leads to liver cancer development. To address this idea, we prepared miR-27a-overexpressing 3T3-L1 adipocytes and cocultured them with HepG2 liver cancer cells. Our results demonstrated that secretory miR-27a promoted liver cancer cell proliferation through the downregulation of the transcription factor FOXO1 and promoted the G1/S cell cycle transition by decreasing the cell cycle inhibitors p21 and p27 and increasing the cell cycle regulator cyclin D1. These findings improve our understanding of the involvement of miR-27a in obesity-liver cancer communication and might provide a novel putative target for obesity-driven primary liver cancer diagnosis and therapy.

Keywords: miR-27a, primary liver cancer, obesity, adipose tissue, FOXO1

\section{Introduction}

Primary liver cancer is the fifth most commonly diagnosed malignancy in men and remains the third leading cause of cancer death in the world. ${ }^{1}$ Liver cancer has been primarily associated with cirrhosis due to chronic infection by the hepatitis $\mathrm{B}$ virus (HBV) and the hepatitis $\mathrm{C}$ virus (HCV), genetic disorders such as hemochromatosis, or toxic injury from excessive alcohol consumption. ${ }^{2}$ Though a substantial number of cases cannot be explained by these etiologies, primary liver cancer is increasingly diagnosed among obese individuals. ${ }^{3}$ In many developed nations, obesity has been recognized as a key component of metabolic syndrome. The accrual of excess adipose tissue during obesity is associated with the dysfunction of this tissue, which can predispose individuals to develop type 2 diabetes mellitus (T2DM) as well as cardiovascular disease. ${ }^{4}$ Moreover, patients with obesity are widely accepted to be at an increased risk of the development and progression of several cancers, including breast, gastrointestinal, renal, esophageal, and reproductive cancers. ${ }^{5-8}$ The increased risk of primary liver cancer that is associated with obesity has been widely reported, this has drawn much attention and, as such, warrants investigation of the key mechanisms that link the obese state with hepatocarcinogenesis.

Obesity-associated primary liver cancer has recently been attributed to molecular mechanisms such as chronic inflammation due to adipose tissue remodeling, ectopic lipid accumulation and lipotoxicity, proinflammatory adipokine secretion altered 
gut microbiota, as well as insulin resistance, which leads to increased levels of insulin and insulin-like growth factors..$^{9,10}$ Adipose tissue within the tumor microenvironment actively contributes to tumor growth and metastasis by functioning as an endocrine organ through the secretion of signaling molecules (such as adipokines, proangiogenic factors, proinflammatory cytokines, and extracellular matrix constituents) and acting as an energy reservoir for embedded cancer cells. ${ }^{11-13}$ Thus, the importance of adipose tissue as an integral contributor to cancer progression is increasingly appreciated. Understanding the key molecular features of obesity-induced dysfunctional adipose tissue might, therefore, offer important mechanistic insights into the relationship between obesity and primary liver cancer.

Intercellular communications are an essential part of the relationship between cells that enable normal cellular function and maintain tissue homeostasis. ${ }^{14}$ As recently as a few years ago, it was believed that RNAs could not behave as extracellular signal molecules because of their vulnerability to the attack of ribonucleases, which largely exist in the body fluid. Evidence showing that microRNAs (miRNAs) are contained in exosomes that are released from mammalian cells and act as signal transducers is presently increasing. ${ }^{15,16}$ MiRNAs are a class of small noncoding RNA (22 nt) that regulate gene expression by binding to the $3^{\prime}$-untranslated region (3'-UTR) of target genes, thus triggering message RNA (mRNA) degradation or protein translation inhibition. ${ }^{17,18}$ Adipocytes have been reported to secrete miRNAs contained in exosomes, which many may regulate the function of distant or neighboring cells during obesity. ${ }^{19}$ The Ogawa group reported that exosomes released from cultured 3T3-L1 adipocytes harbored 143 miRNAs, most of which are adipocyte-specific and reflect the abundance of their expression levels in the donor cells. ${ }^{20}$ Based on these observations, we reasoned that the miRNAs excreted from adipose tissue could be increased in obesity, leading to the increased stimulation of liver cancer development.

To address these issues, we first studied miR-27a expression using tumor tissue, adipose tissue, and plasma samples from obese liver cancer patients, nonobese liver cancer patients, and healthy individuals. Next, we transfected 3T3-L1 cells with a miR-27a overexpression plasmid and investigated whether miR-27a released from adipose cells transfers proliferation signals to liver cancer cells in a coculture experiment. Our results test the hypotheses that miR-27a, which is characteristically overexpressed and secreted by adipose tissue during obesity, is a potent regulator of liver cancer proliferation by targeting the forkhead transcription factor 1 (FOXO1).

\section{Materials and methods Clinical samples}

Thirty paired liver cancer tissue samples $(\mathrm{T})$ compared with matched adjacent normal tissue (ANT) samples were collected during routine therapeutic surgery of nonobese $(n=15)$ and obese $(n=15)$ hepatocellular carcinoma (HCC) patients in our hospital. Written informed consent was obtained from each patient. Omental adipose tissues were also obtained from the same patient. This work was approved by the Ethics Committee of China-Japan Union Hospital of Jilin University. All tissues were immediately frozen in liquid nitrogen after explantation. Plasma samples from healthy people (controls, $n=10$ ) were collected from our department. People without any current clinical conditions and without a family history of diseases such as cancer, HIV, diabetes, and autoimmune diseases were qualified as healthy individuals. Body mass index (BMI) was calculated as weight divided by height in meter squared.

\section{Cell culture and transfection}

The mouse embryo 3T3-L1 cell line and the human liver cancer HepG 2 cells were obtained from the American Type Culture Collection (ATCC, Manassas, VA, USA). The cells were grown in Dulbecco's Modified Eagle's Medium (DMEM) (Gibco, Carlsbad, CA, USA) with high glucose content, supplemented with 10\% fetal bovine serum (FBS) (Gibco), 100 units $/ \mathrm{mL}$ penicillin, and $100 \mu \mathrm{g} / \mathrm{mL}$ streptomycin. All of the cells were incubated at $37^{\circ} \mathrm{C}$ in a humidified chamber containing $5 \% \mathrm{CO}_{2}$. The miR-27a overexpression plasmid and the control vector were from GeneCopoeia (Rockville, MD, USA). To establish stable miR-27a-overexpressing $3 \mathrm{~T} 3-\mathrm{L} 1$ cell lines, approximately $1 \times 10^{6}$ cells were seeded and cultured in $35 \mathrm{~mm}$ cell culture dishes. When the cells reached $90 \%-95 \%$ confluence, $50 \mu \mathrm{g}$ of the miR-27a plasmid was transfected with Lipofectamine 2000 (Invitrogen Life Technologies, Waltham, MA, USA) according to the manufacturer's instructions. The transfected cells were incubated at $5 \% \mathrm{CO}_{2}$ and $37^{\circ} \mathrm{C}$. Four hours later, the transfection media was changed to DMEM containing 10\% FBS. Twelve hours after transfection, the cells were replated in a $10-\mathrm{cm}$ dish followed by a 3 -week selection with antibiotic. Ten surviving single colonies were picked up from each transfectant and then cultured for another 2 weeks. The cells expressing the largest amount of miR-27a among the transfectants were used as miR-27a stably expressing cells. 


\section{miRNA isolation and quantitative real- time polymerase chain reaction}

Total RNA and miR fractions were isolated from tissue samples, serum, and HepG2 cell lines with TRIzol reagent (Invitrogen). RNA was reverse transcribed into cDNA with TransScript First-Strand cDNA synthesis supermix (TransScript, Invitrogen), following the manufacturer's instructions. MicroRNA extraction was performed using the miRNA Extraction Kit (Tiangen, Beijing, People's Republic of China). Quantitative real-time polymerase chain reaction (qRT-PCR) was performed using a FastStart Universal STBR Green Master (Rox) from Roche (Basel, Switzerland). Primers of miR-27a and U6 were obtained from Ribobio (Guangzhou, People's Republic of China). The expression of miR-27a was normalized with U6 using the $2^{-\Delta \Delta \mathrm{CT}}$ method. The miRNA expression levels were defined based on the threshold cycle $(\mathrm{Ct})$, and the relative expression levels were calculated as 2-[(Ct of miR-27a)-(Ct of U6)].

RT-PCR was performed using the Applied Biosystems 7,300 Sequence Detection system using the following primers for $\mathrm{FOXO1}$, (forward, 5'-ACATTTCGTCCTCGAACCAGCTCA-3' and reverse, 5'-ATTTCAGACAGACTGGGCAGCGTA-3'); p21, (forward, 5'-CGATGCCAACCTCCTCAACGA-3' and reverse, 5'-TCGCAGACCTCCAGCATCCA-3'); p27 (forward, 5'-TGCAACCGACGATTCTTCTACTCAA-3' and reverse, 5'-CAAGCAGTGATGTATCTGAT AAACAAGGA-3'); and cyclin D1 mRNA (forward, 5'-AACTACCTGGACCGCTTCCT-3' and reverse, 5'-CCACTTGAGCTTGTTCACCA-3'). The expression data were normalized to the geometric mean expression level of the house keeping gene GAPDH (forward, 5'-GACTCATGACCACAGTCCATGC-3' and reverse, 3'-AGAGGCAGGGATGATGTTCTG-5') and calculated using

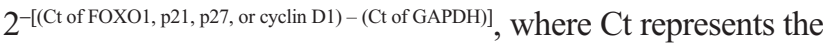
threshold cycle for each transcript.

\section{Coculture experiment and MTT assay}

miR-27a inhibitors and mimics were obtained from RiboBio. In coculture experiments, $2 \times 10^{5}$ cells/well of miR-27a-overexpressed-3T3-L1 cells were plated in 6-well plates. After 12 hours, $1 \times 10^{5}$ HepG 2 cells were seeded in transwell and cocultured with miR-27a-overexpressed3T3-L1 cells at $37^{\circ} \mathrm{C}$. To the medium, $100 \mathrm{nM}$ miR-27a inhibitor was added. After coculture for 24 or 48 hours, cell medium was collected, and the cells were incubated in $0.1 \mathrm{mg} / \mathrm{mL} \mathrm{MTT}$ at $37^{\circ} \mathrm{C}$ for 3 hours and lysed in dimethyl sulfoxide (DMSO) at room temperature for 30 minutes.
The absorbance in each well was measured at $570 \mathrm{~nm}$ using a microplate reader. Each experiment was performed in triplicate. The survival rate of cells was expressed as A/B 100\%, where A was the absorbance value from the experimental cells and B was that from the control cells.

\section{Cell cycle analysis}

Cells were collected by trypsinization, washed in ice-cold PBS, and fixed in $80 \%$ ice-cold ethanol in PBS. Before staining, the cells were pelleted by centrifugation at $4^{\circ} \mathrm{C}$ and resuspended in chilled PBS, followed by incubation with $20 \mu \mathrm{g} / \mathrm{mL}$ of propidium iodide (Sigma-Aldrich, St Louis, MO, USA) for 20 minutes at room temperature. Fifty thousand cells per group were analyzed on a flow cytometer (FACS Calibur; BD Biosciences, San Jose, CA, USA).

\section{Western blot analysis}

Cells were harvested and lysed with ice-cold lysis buffer containing $50 \mathrm{mM}$ Tris-HCl, pH 6.8, 100 mM 2-mercaptoethanol, $2 \% \mathrm{w} / \mathrm{v}$ sodium dodecyl sulfate, and 10\% glycerol. After centrifugation, the supernatants were quantified and separated by $10 \%$ SDS-PAGE (sodium dodecyl sulfate-polyacrylamide gelelectrophoresis) and transferred to a nitrocellulose membrane (Amersham Bioscience, Buckinghamshire, UK). After blocking with $10 \%$ nonfat milk in Tris-buffer saline (TBS), membranes were immunoblotted with antibodies, followed by HRP-linked secondary antibodies. Detection of antibody binding was performed using the enhanced chemiluminescence kit (Amersham Pharmacia Biotech, Amersham, UK). All western blot analyses were repeated three times. Anti-FOXO1, anti-cyclin D1, anti-p27, and anti-p21 were purchased from Santa Cruz Biotechnologies, Inc. (Santa Cruz, CA, USA). Protein levels were normalized to total GAPDH, using a rabbit polyclonal anti-GAPDH antibody (Santa Cruz Biotechnologies, Inc.).

\section{Immunohistochemistry}

Immunohistochemical staining for FOXO1, p21, p27, and cyclin D1 in the above-mentioned 30 HCC samples was performed following the methods described previously. Briefly, after deparaffinization and blocking, the sections were incubated overnight at $4{ }^{\circ} \mathrm{C}$ with the antibody. The sections were counterstained with Meyer's hematoxylin. FOXO1, p21, p27, and cyclin D1-positive cells were defined as those with brown staining in the nucleus and cytoplasm, respectively. Signals in tumor tissue and matched ANT were visually quantified using the same scoring system (0-9), multiplied intensity of signal, and percentage of positive 
cells ( signal: $0=$ no signal, $1=$ weak signal, $2=$ intermediate signal, and $3=$ strong signal; percentage: $0=0 \%, 1=<25 \%$, $2=25 \%-50 \%$, and $3=>50 \%$ ).

\section{Statistical analysis}

All experiments were completed at least three times unless otherwise indicated. Data are expressed as the means \pm standard deviations. The two-tailed Student's $t$-test was used to evaluate the significance of the difference between two groups in all pertinent experiments. Differences with $P<0.05$ were considered to be statistically significant.

\section{Results}

miR-27a is upregulated in liver cancer patients who are obese

To investigate the role of miR-27a in liver cancer patients who are obese, we first compared its expression levels between clinical liver cancer tissues (T) and matched ANT from 15 cases of obese patients and 15 cases of nonobese patients. By qRT-PCR, we showed that the miR-27a levels were significantly higher in liver cancer samples than in the nontumor liver samples from all liver cancer patients (Figure 1A). Interestingly, the overall expression level of miR-27a in

\section{A}
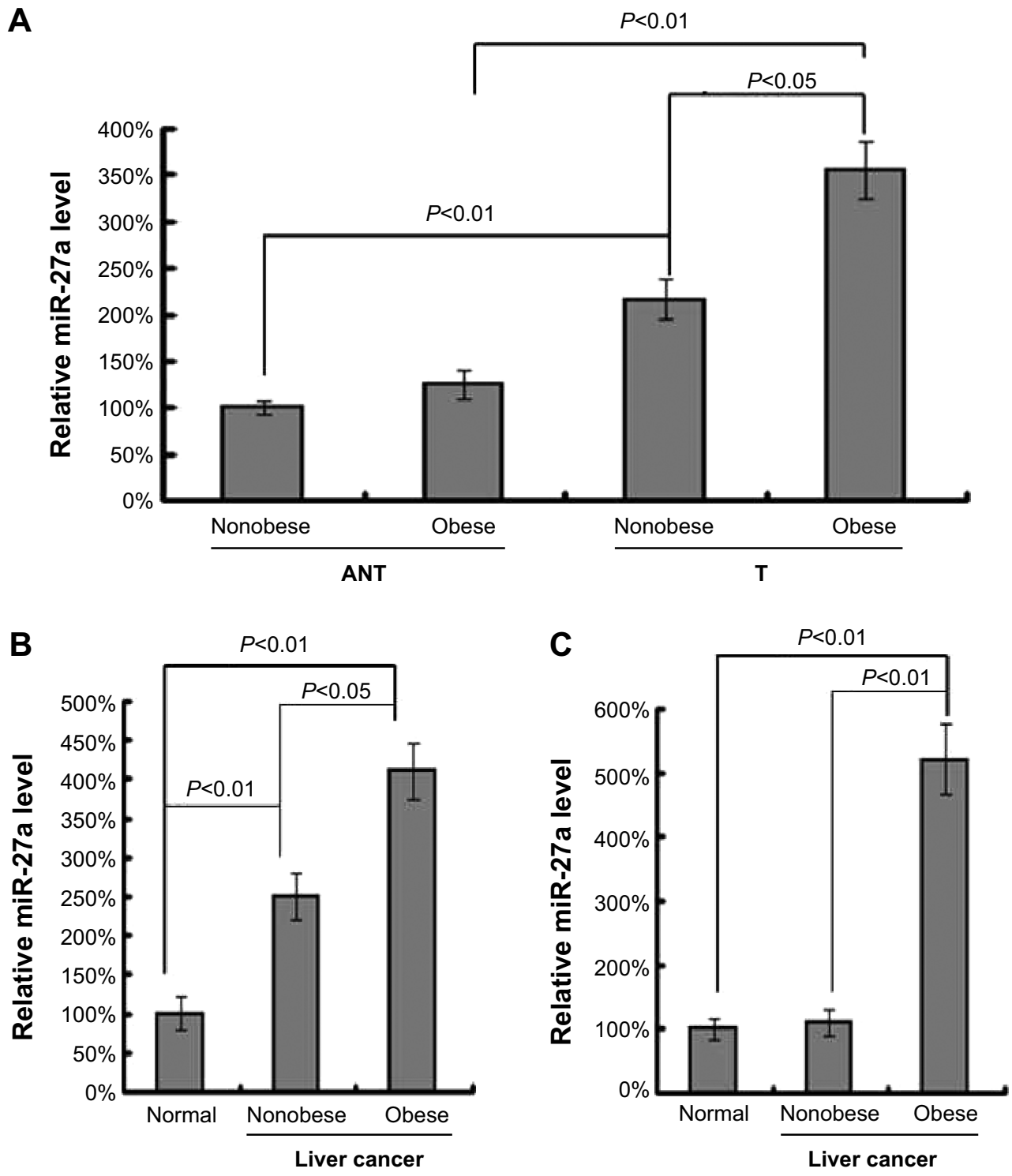

Figure I miR-27a is upregulated in tumor tissue, serum and adipose tissue of obese liver cancer patients.

Notes: (A) qRT-PCR analysis of miR-27a expression in liver cancer tissue (T) compared with matched ANT from obese patients $(n=15)$ and nonobese patients ( $n=15)$. (B) qRT-PCR analysis of miR-27a expression in plasma samples from obese patients ( $n=15)$, nonobese patients ( $n=15)$, and healthy individuals (controls, $n=10)$. (C) qRT-PCR analysis of miR-27a expression in omental adipose tissue from obese patients $(n=15)$, nonobese patients $(n=15)$, and healthy individuals $($ controls, $n=15)$. Each bar represents the mean $\pm S D$ of three independent experiments.

Abbreviations: qRT-PCR, quantitative real-time polymerase chain reaction; T, tissue; ANT, adjacent normal tissue; SD, standard deviation. 
cancer samples was increased about two-fold in obese patients rather than nonobese patients, indicating that upregulated miR-27a might be a biomarker in obesity-induced liver cancer. Next we analyzed the serum levels of miR-27a among obese liver cancer patients, non obese liver cancer patients, and healthy individuals. The results (Figure 1B) showed that the serum level of miR-27a was highly upregulated in obese liver cancer patients compared with both nonobese liver cancer patients and healthy individuals. Recent studies have reported that a panel of miRNAs was significantly dysregulated in an in-depth analysis of miRNomes in omental adipose tissue of T2DM patients, and miR-27a was identified as one of these miRNAs. ${ }^{19}$ We further detected the expression of miR-27a in omental adipose tissue samples from obese liver cancer patients, nonobese liver cancer patients, and healthy individuals. As shown in Figure 1C, the miR-27a levels in omental adipose tissue were significantly increased in obese liver cancer patients by approximately five times compared with those of nonobese liver cancer patients and healthy individuals. Taken together, these results indicated that miR-27a is upregulated in obese liver cancer patients, which might play a potential role in defining liver cancer development and mediating the obesity-cancer communication.

\section{Secretory miR-27a promotes liver cancer cell proliferation through FOXOI}

To test our hypothesis, we conducted experiments to examine whether 3T3-L1 cells overexpressing miR-27a could stimulate the growth of liver cancer cells. We first established and confirmed stable miR-27a-overexpressed 3T3-L1 cell lines (Figure 2A). Cultured 3T3-L1 cells transfected with a miR-27a overexpression plasmid showed a significantly increased level in the cells and media, indicating that 3T3-L1 cells stably overexpressing miR-27a secreted a large of miR$27 \mathrm{a}$ into media. After 24 or 48 hours of incubation with the miR-27a-overproducing 3T3-L1 cells and control 3T3-L1 cells, we observed that HepG2 cells cocultured with miR27a-overproducing 3 T3-L1 cells showed a dramatic increase in proliferation as measured by MTT assay (Figure 2B). Importantly, the increased cell viability was recovered by the addition of a miR-27a inhibitor in the media. Moreover, we detected the miR-27a level in HepG2 cells and media at 24 and 48 hours in these coculture experiments. As shown in Figure 2C and D, the miR-27a levels in HepG2 cells and media were significantly enhanced in miR-27a-overproducing 3T3-L1 cells compared with the control group at 24 or 48 hours, whereas the addition of a miR-27a inhibitor blocked the
miR-27a levels in the cells and media. These data indicated that the proliferation of HepG2 cells is attributable to the secretory miR-27a contained in the supernatant of miR-27aoverexpressed 3T3-L1 cells.

Because secretory miR-27a significantly affected cell proliferation in HepG2 cells, we hypothesized that miR-27a could function by affecting the cell cycle of liver cancer cells. We tested this hypothesis by flow cytometry. The results revealed that secretory miR-27a drastically decreased the percentage of cells in the $\mathrm{G}_{1} / \mathrm{G}_{0}$ peak and increased the percentage of cells in the $\mathrm{S}$ peak in HepG2 cells compared with control cells at 48 hours (Figure 3A). However, the addition of a miR-27a inhibitor dramatically increased the number of cells in the $\mathrm{G}_{0} / \mathrm{G}_{1}$ peak and decreased those in the $\mathrm{S}$ peak. Thus, secretory miR-27a may enhance the proliferation of liver cancer cells by promoting the $\mathrm{G}_{1} / \mathrm{S}$ cell cycle transition. FOXO1 is a putative tumor suppressor, and the expression of this gene is dysregulated in some cancers, including liver, breast, prostate, and endometrial cancers. Guttilla et $\mathrm{al}^{21}$ first reported that miR-27a directly targets various regions of the $3^{\prime}$-UTR to repress the endogenous expression of FOXO1 in breast cancer. To further study miR-27a transfer on a molecular level, we performed a target gene expression analysis of FOXO1. As predicted, western blotting revealed that the ectopic expression of miR-27a in HepG2 cells decreased the expression of FOXO1 proteins, whereas the addition of a miR-27a inhibitor increased its expression (Figure 3C and D). We also observed a similar result for the FOXO1 mRNA levels (Figure 3B). These data indicated that secretory miR-27a led to the decreasing of FOXO1 expression at the transcriptional and translational levels. The FOXO1 transcription factor orchestrates the regulation of genes involved in the cell cycle check-points, apoptotic response, and cellular metabolism. FOXO1 can regulate a series of genes that are relevant to the cell cycle at a transcriptional level, including p21, p27, and the CDK regulator cyclin D1. Using western blotting and qRT-PCR analysis, we observed that $\mathrm{p} 21$ and $\mathrm{p} 27$ protein and mRNA levels were downregulated and cyclin D1 protein and mRNA levels were upregulated in overexpressed miR-27a-3T3-L1 cocultured HepG2 cells compared with control cells (Figure 3B-D). However, the addition of a miR-27a inhibitor reversed this shift. Taken together, these results demonstrated that secretory miR-27a promoted liver cancer cell proliferation through the downregulation of the transcription factor FOXO1 and finally promoted the $\mathrm{G}_{1} / \mathrm{S}$ cell cycle transition by decreasing the cell cycle inhibitors p21 and p27, as well as increasing cell cycle regulator cyclin D1. 
A

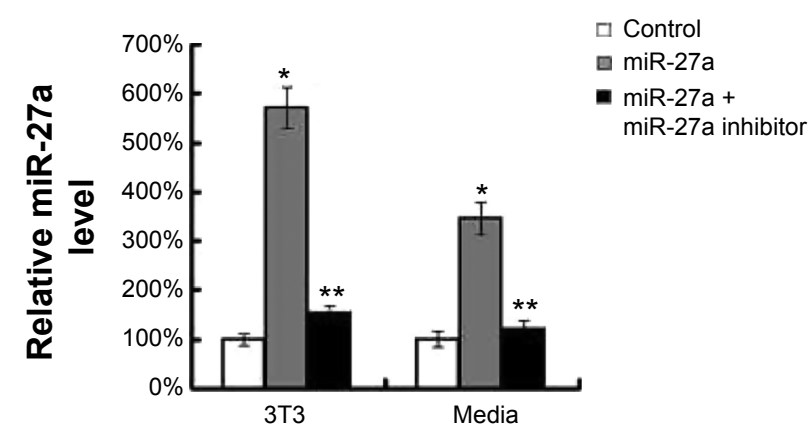

C

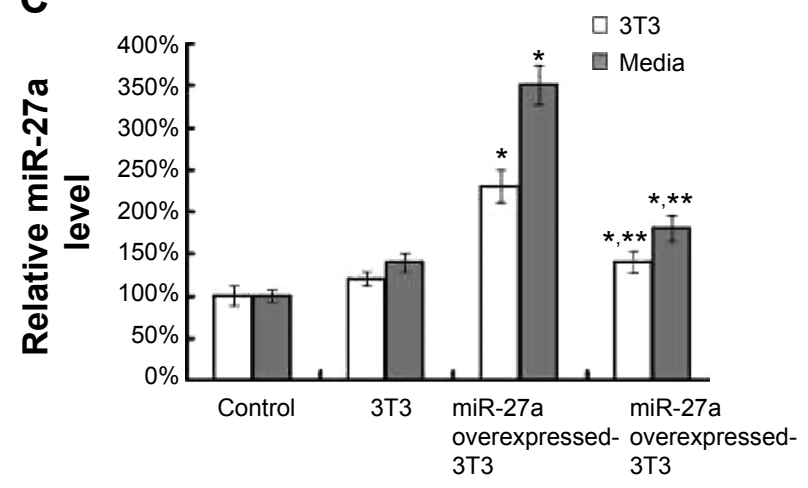

$\operatorname{miR}-27 a$

inhibitor
B

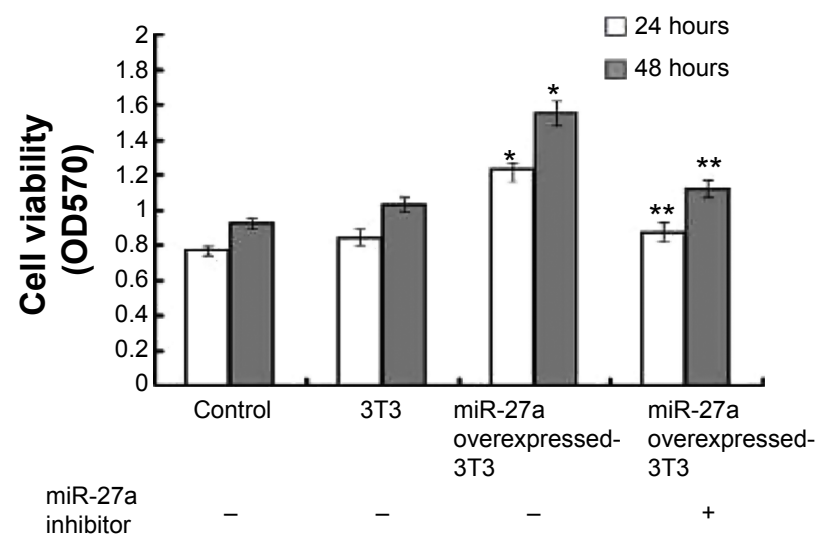

D

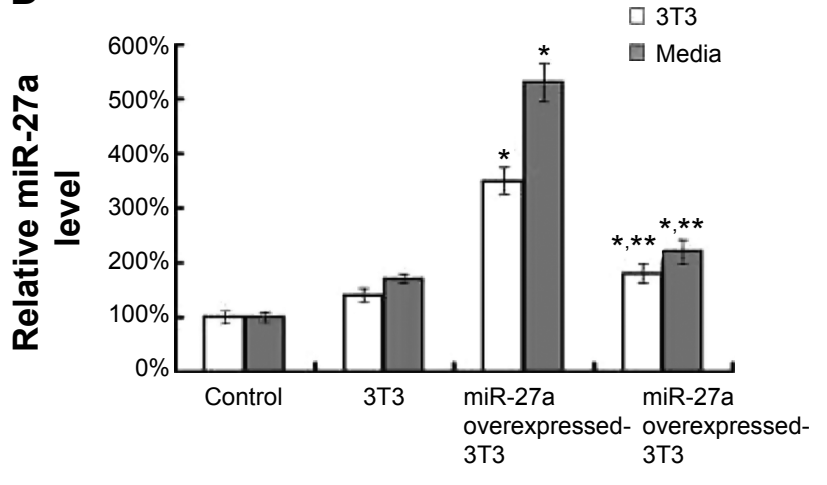

miR-27a

inhibitor

Figure 2 Secretory miR-27a promotes liver cancer cell proliferation.

Notes: (A) qRT-PCR analysis of miR-27a expression in miR-27a overexpressing 3T3-LI cells and cell culture media. (B) Cell viability as measured by MTT assay of HepG2 cells under cocultured alone, with 3T3-LI, miR-27a overexpressing 3T3-LI and miR-27a overexpressing 3T3-LI plus a miR-27a inhibitor for 24 or 48 hours. (C, D) qRT-PCR analysis of miR-27a expression in HepG2 cells alone, under cocultured with 3T3-LI, miR-27a overexpressing 3T3-LI and miR-27a overexpressing 3T3-LI plus a miR-27a inhibitor for 24 (C) or 48 (D) hours. Each bar represents the mean \pm SD of three independent experiments. $* P<0.05$ compared with control, $* * P<0.05$ compared with miR-27a overexpressed 3T3-LI group.

Abbreviations: qRT-PCR, quantitative real-time polymerase chain reaction; SD, standard deviation.

\section{miR-27a expression is correlated with FOXOI in obese liver cancer patients}

To further investigate this proposed regulation in liver cancer samples, we measured miR-27a using qRT-PCR analysis and performed immunohistochemical staining for FOXO1, p21, p27, and cyclin D1 on the paraffin-embedded cancer tissues ( $T$ ) compared with matched ANT from 15 obese patients and 15 nonobese patients. As shown in Figures 1, 4, and 5, tumor tissues with high miR-27a expression exhibited low levels of FOXO1, p21, and p27 and high levels of cyclin D1. Additionally, matched ANTs with low miR27a expression showed high levels of FOXO1, p21, and p27 and low levels of cyclin D1, revealing a strong link between miR-27a and proliferative potential. Remarkably, consistent with our in vitro experiments, higher miR-27a expression of obese liver cancer patients was significantly correlated with lower levels of FOXO1, p21, and p27 and higher levels of cyclin D1 compared with nonobese liver cancer patients with lower miR-27a expression. Collectively, these results strongly indicated that miR-27a expression is associated with liver cancer progression and prognosis, acting as a potential oncogenic biomarker involved in obesity-cancer communication.

\section{Discussion and conclusion}

Although the role of genetic alterations in oncogenes and tumor suppressor genes has been extensively studied, epigenetic mechanisms contributing to liver cancer development are less well characterized. The influence of miRNA on obesity-cancer communication is becoming increasingly recognized. In this study, we clearly demonstrated that miR-27a is more highly upregulated in cancer, plasma, and adipose samples from obese-liver cancer cases than nonobese liver cancer cases, and we reasoned that miR-27a excreted 
A
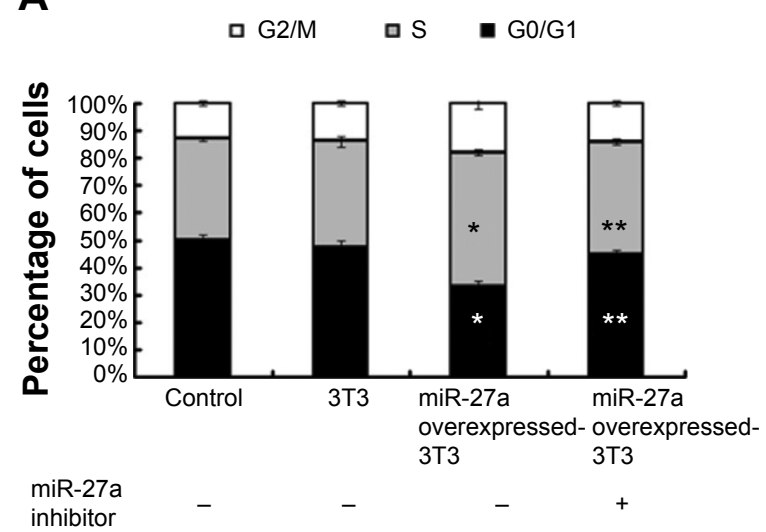

C

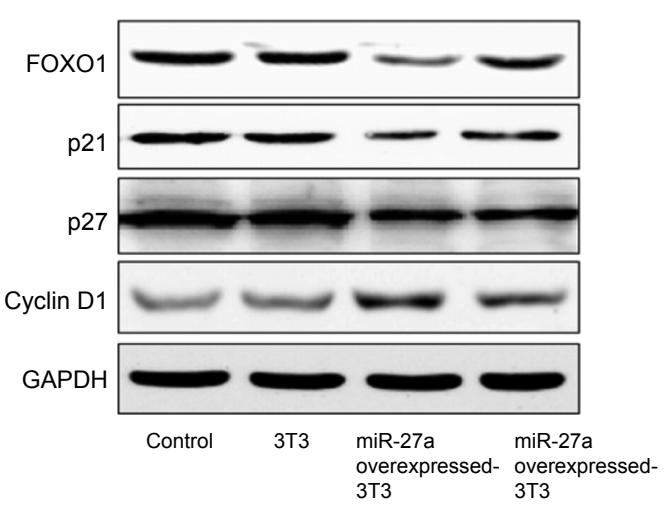

miR-27a

inhibitor
3T3

$+$
B

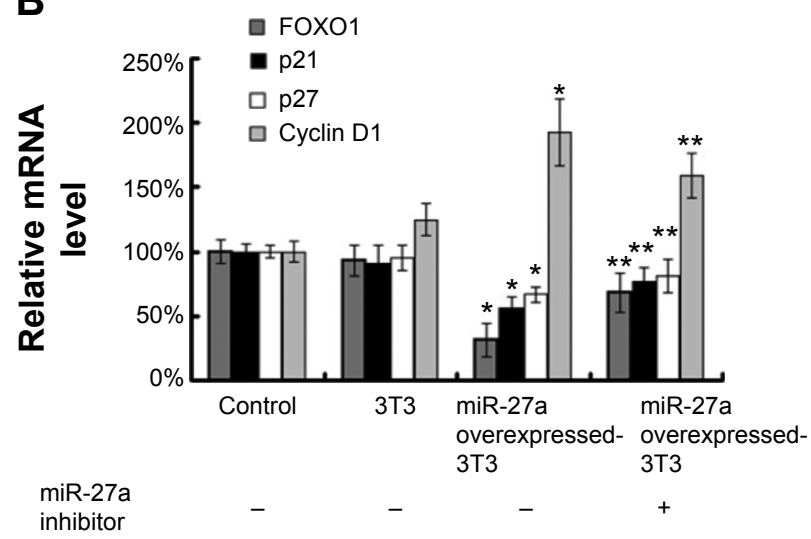

D

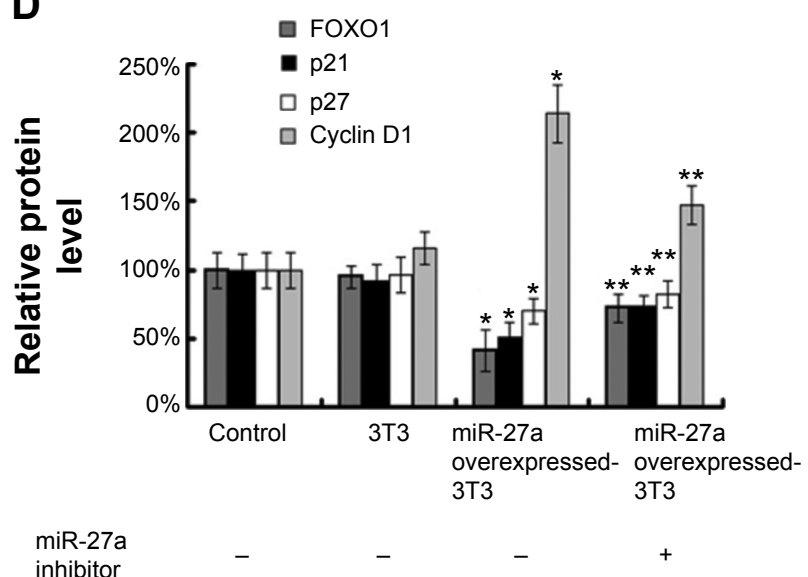

Figure 3 Secretory miR-27a induced liver cancer cell GI/S transition by targeting FOXOI.

Notes: (A) Flow cytometric analysis of the cell cycle of HepG2 cells alone, under cocultured with 3T3-LI, miR-27a overexpressing 3T3-LI and miR-27a overexpressing 3T3LI plus miR-27a inhibitor for 48 hours. (B) qRT-PCR analysis of FOXOI, p2I, p27, and cyclin DI mRNA expression in HepG2 cells alone, under cocultured with 3T3-LI, miR27a overexpressing 3T3-LI and miR-27a overexpressing 3T3-LI plus miR-27a inhibitor for 48 hours. (C-D) Western blot analysis of FOXOI, p2I, p27, and cyclin DI protein expression in HepG2 cells alone, under cocultured with 3T3-LI, miR-27a overexpressing 3T3-LI and miR-27a overexpressing 3T3-LI plus miR-27a inhibitor for 48 hours. Each bar represents the mean \pm SD of three independent experiments. $* P<0.05$ compared with control, $* * P<0.05$ compared with miR-27a overexpressed $3 T 3$-LI group. Abbreviations: qRT-PCR, quantitative real-time polymerase chain reaction; FOXOI, forkhead box OI; p2I, cyclin-dependent kinase inhibitor IA; p27, cyclin-dependent kinase inhibitor IB; SD, standard deviation.

from adipose tissue leaded to liver cancer development. It is well established that adipose tissue secretes a wide array of molecules. ${ }^{22}$ These adipokines, which are exclusively or partially secreted by adipocytes or stromal-vascular fraction cells, are likely to have a role in modulating the risk of cancer progression. ${ }^{23}$ Few studies examined the effect of adipocytes in liver cancer cell growth. In this study, we prepared miR27a-overexpressing 3T3-L1 adipocytes and cocultured them with HepG2 liver cancer cells. Our study documented that the miR-27a derived from 3T3-L1 adipocytes had the ability to promote liver cancer cell proliferation.

If the movement of miR-27a is critical for liver cancer development, the transfer of silencing signals should be illustrated. MiR-27a is located on chromosome 19 and is overexpressed in liver cancer, breast cancer, gastric cancer, lung cancer, colorectal cancer, and pancreatic cancer. ${ }^{21,24-28}$ The increased expression of miR-27a was shown to promote tumor development by targeting multiple cellular factors, including FOXO1, ZBTB10, and Myt-1. 21,27,29 All of these studies support the oncogenic role of miR-27a. Human Forkhead box O (FOXO) proteins (FOXO1, FOXO3a, FOXO4, and FOXO6) are key effectors of PI3K/Akt signaling and regulate many biological processes, such as cell cycle regulation, cell differentiation, tumorigenesis, and oxidative stress responses. ${ }^{30-32}$ The FOXO1 levels were higher in HCC patients with better prognosis ( $>3$-year survival) than in those with poor prognosis..$^{33,34}$ Therefore, FOXO1 repression in $\mathrm{HCC}$ is likely to be associated with an unfavorable outcome. In this study, secretory miR-27a increased liver cancer cell proliferation through delay of the $\mathrm{G}_{1} / \mathrm{S}$ transition and decreased FOXO1 protein expression, which correlated with reduced expression of the genes regulated by FOXO1, including the cell-cycle inhibitors p21 and p27, and upregulation of the cell-cycle regulator 


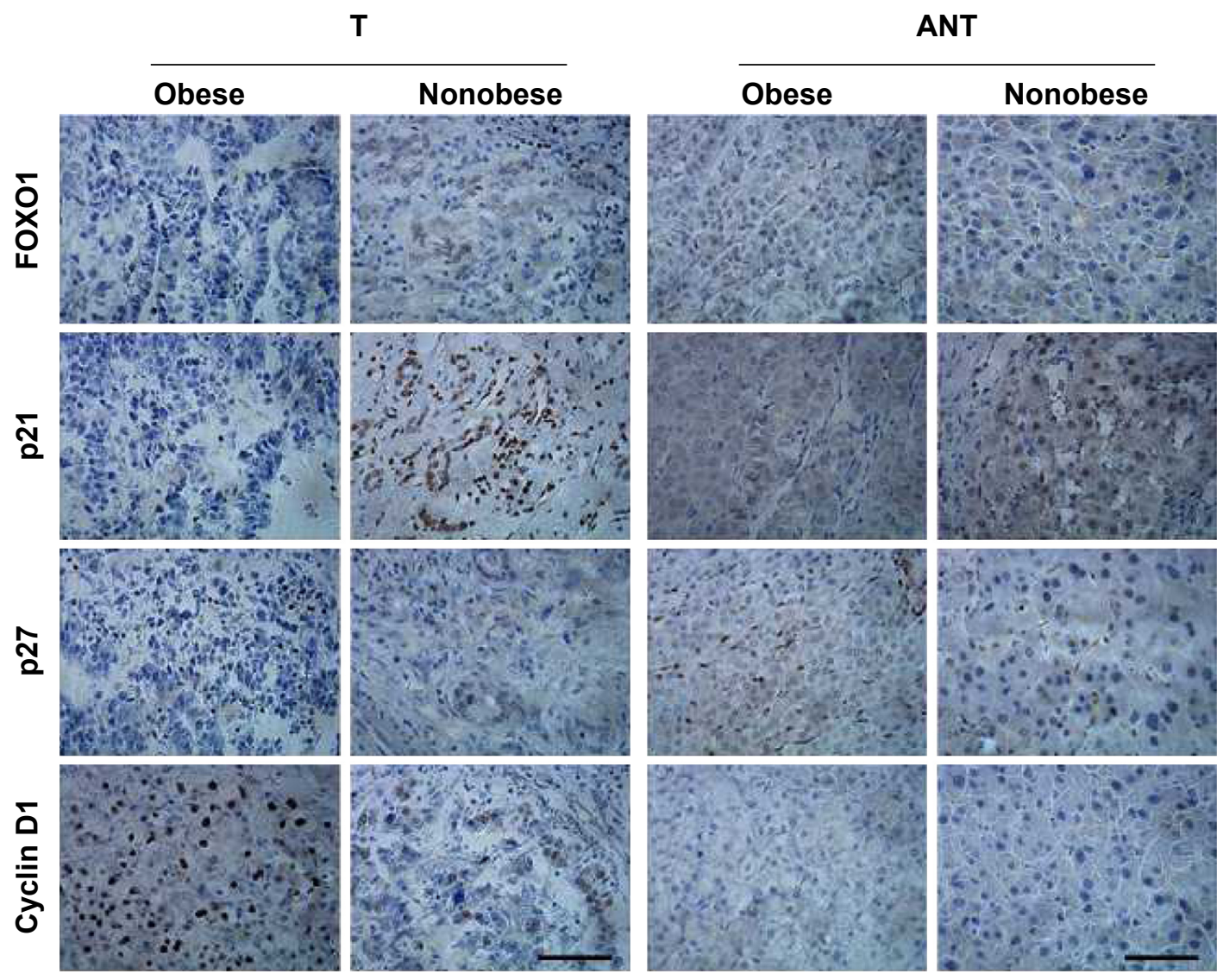

Figure 4 Immunohistochemical staining of FOXOI, p2I, p27, and cyclin DI in obese liver cancer patients.

Notes: Representative images of immunohistochemical staining for FOXOI, p2I, p27, and cyclin DI in liver cancer tissue (T) compared with matched ANT from obese cases and nonobese cases. Scale bar represents $100 \mu \mathrm{m}$.

Abbreviations: FOXOI, forkhead box OI; p2I, cyclin-dependent kinase inhibitor IA; p27, cyclin-dependent kinase inhibitor IB; T, tissue; ANT, adjacent normal tissue.

cyclin D1. In agreement with our results, the downregulation of FOXO1 in human breast cancer cells can promote transformation and tumor progression..$^{35}$ Moreover, it has been demonstrated that FOXO1 induces $\mathrm{G}_{1}$ phase cell-cycle arrest in glioma cells and renal cell carcinoma due to inhibition of tumor suppressor phosphatase and tensin homolog deleted on chromosome ten (PTEN), via the upregulation of $\mathrm{p} 27 .^{36,37}$

There are several potential mediators of obesity-related HCC that were discussed in previous reviews, such as lipotoxicity, changes in the gut microbiome, an imbalance in proinflammatory and anti-inflammatory cytokines, reduced adiponectin secretion, and increased leptin and stimulation of the insulin-like growth factor 1 (IGF-1) axis by hyperinsulinemia. ${ }^{9}$ These biomarkers may also explain the joint effect of obesity, with the traditional risk factors further increasing the risk of liver cancer. However, only a few prospective epidemiological studies have examined the association between inflammatory or metabolic biomarkers and risk of liver cancer in a general population. Having this information is important because evidence of the relation between obesity-related biomarkers and the risk of liver cancer may provide clues for understanding the underlying etiological mechanisms. In our study, we observed that the high miR-27a levels in obese liver cancer cases induced more liver cancer proliferation compared with nonobese liver cancer cases, indicating that miR-27a might become a potential biomarker for liver cancer diagnosis and prognosis. The main limitation of this study was the relatively small number of cases, which inevitably limited the precision of our estimates of relative risk between obesity and liver cancer. Therefore, the clinical significance and mechanism of miR-27a upregulation in obese liver cancer require further investigation.

\section{Conclusion}

Our current study demonstrates that obese adipose tissuesecreted miR-27a is upregulated in obese liver cancer 
A

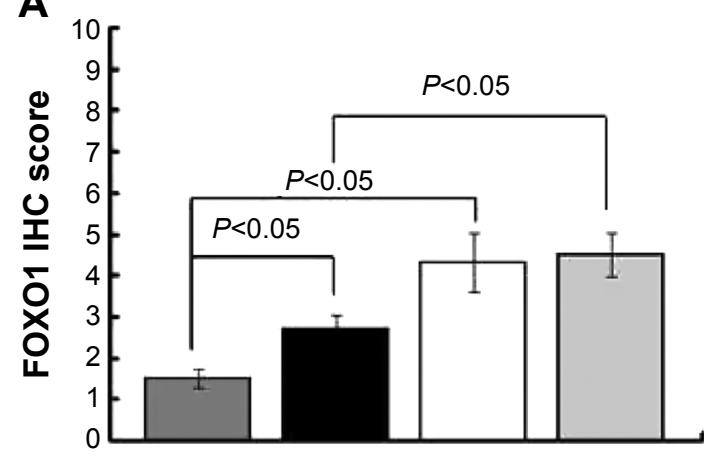

C

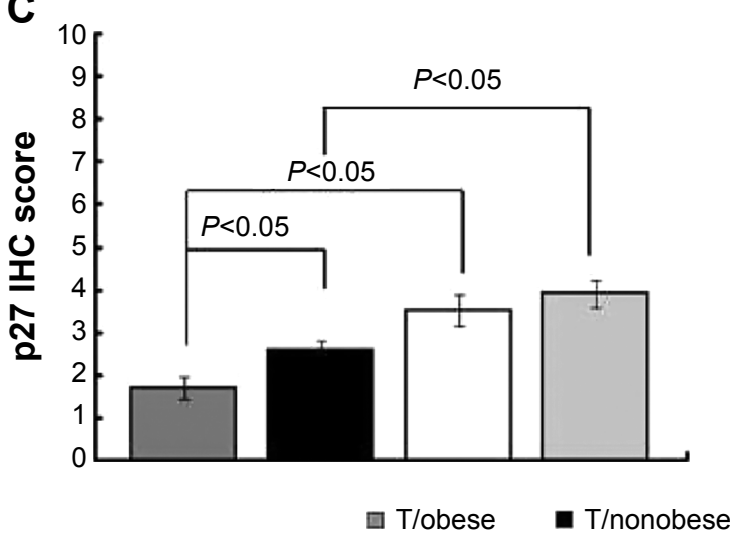

B

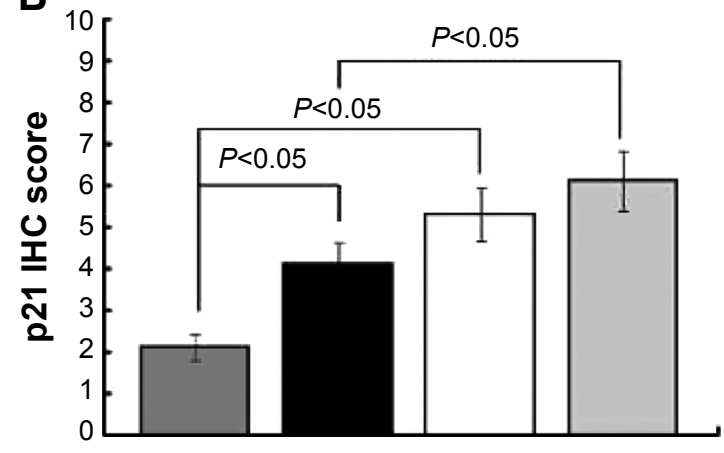

D

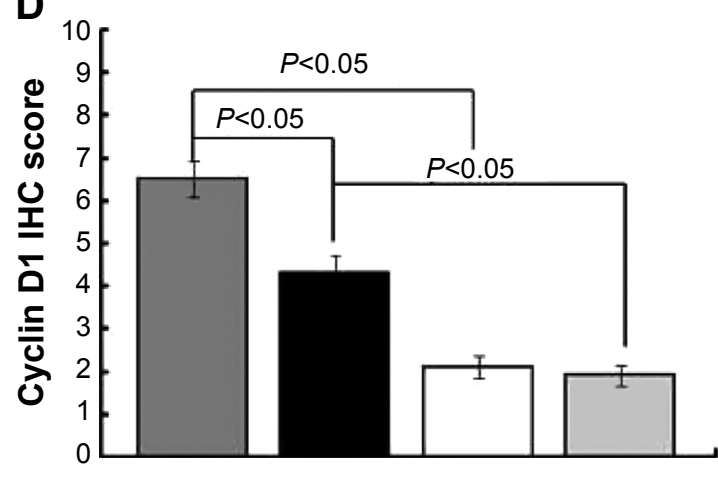

$\square$ ANT/obese $\quad \square$ ANT/nonobese

Figure 5 miR-27a expression is correlated with FOXOI in obese liver cancer patients.

Notes: Bar charts show the association between miR-27a expression and FOXOI (A), p2I (B), p27 (C), and cyclin DI (D) expression, as indicated by immunohistochemical staining. Error bars represent \pm SD.

Abbreviations: FOXOI, forkhead box OI; p2I, cyclin-dependent kinase inhibitor IA; p27, cyclin-dependent kinase inhibitor IB; SD, standard deviation; T, tissue; ANT, adjacent normal tissue.

tissues. Cocultured with miR-27a-overexpressing-3T3-L1 adipocytes drastically promotes liver cancer cell proliferation through the downregulation of the transcription factor FOXO1, promoting the $\mathrm{G}_{1} / \mathrm{S}$ cell cycle transition by decreasing the cell cycle inhibitors p 21 and p 27 and increasing the cell cycle regulator cyclin D1. These findings improve our understanding of the involvement of miR-27a in obesity-liver cancer communication and might provide a novel putative target for obese liver cancer diagnosis and therapy.

\section{Acknowledgments}

We thank Tissue Bank of China-Japan Union Hospital, Jilin University, for collection of the specimens used in this study. This study was supported by research grants from the Nature Science Foundation of Jilin Province, China (201215079).

\section{Disclosure}

The authors report no conflicts of interest in this work.

\section{References}

1. Maluccio M, Covey A. Recent progress in understanding, diagnosing, and treating hepatocellular carcinoma. CA Cancer J Clin. 2012;62:394-399.

2. Ding J, Wang HY. Multiple interactive factors in hepatocarcinogenesis. Cancer Lett. 2014;346:17-23.

3. Larsson SC, Wolk A. Overweight, obesity and risk of liver cancer: a meta-analysis of cohort studies. Br J Cancer 2007;97:1005-1008.

4. Sun K, Kusminski CM, Scherer PE. Adipose tissue remodeling and obesity. J Clin Invest. 2011;121:2094-2101.

5. Vucenik I, Stains JP. Obesity and cancer risk: evidence, mechanisms, and recommendations. Ann NY Acad Sci. 2012;1271:37-43.

6. Sinicrope FA, Dannenberg AJ. Obesity and breast cancer prognosis: weight of the evidence. J Clin Oncol. 2011;29:4-7.

7. Gati A, Kouidhi S, Marrakchi R, et al. Obesity and renal cancer role of adipokines in the tumor-immune system conflict. Oncoimmunology. 2014;3(1):e27810.

8. Ryan AM, Duong M, Healy L, et al. Obesity, metabolic syndrome and esophageal adenocarcinoma: epidemiology, etiology and new targets. Cancer Epidemiol. 2011;35:309-319.

9. El-Serag HB, Kanwal F. Obesity and hepatocellular carcinoma: hype and reality. Hepatology. 2014;60:779-781.

10. Karagozian R, Derdak Z, Baffy G. Obesity-associated mechanisms of hepatocarcinogenesis. Metabolism. 2014;63:607-617.

11. Park J, Euhus DM, Scherer PE. Paracrine and endocrine effects of adipose tissue on cancer development and progression. Endocr Rev. 2011;32:550-570 
12. Prieto-Hontoria PL, Perez-Matute P, Fernandez-Galilea M, Bustos M, Martinez JA, Moreno-Aliaga MJ. Role of obesity-associated dysfunctional adipose tissue in cancer: a molecular nutrition approach. Biochim Biophys Acta. 2011;1807:664-678.

13. Sun BC, Karin M. Obesity, inflammation, and liver cancer. J Hepatol. 2012;56:704-713.

14. Mittelbrunn M, Sanchez-Madrid F. Intercellular communication: diverse structures for exchange of genetic information. Nat Rev Mol Cell Biol. 2012;13:328-335.

15. Keller S, Ridinger J, Rupp AK, Janssen JW, Altevogt P. Body fluid derived exosomes as a novel template for clinical diagnostics. J Transl Med. 2011;9:86.

16. Kosaka N, Iguchi H, Yoshioka Y, Takeshita F, Matsuki Y, Ochiya T. Secretory mechanisms and intercellular transfer of microRNAs in living cells. J Biol Chem. 2010;285:17442-17452.

17. Calin GA, Croce CM. MicroRNA signatures in human cancers. Nat Rev Cancer. 2006;6:857-866.

18. Ameres SL, Zamore PD. Diversifying microRNA sequence and function. Nat Rev Mol Cell Biol. 2013;14:475-488.

19. Kloting N, Berthold S, Kovacs P, et al. MicroRNA expression in human omental and subcutaneous adipose tissue. PLoS One. 2009;4: e4699.

20. Ogawa R, Tanaka C, Sato M, et al. Adipocyte-derived microvesicles contain RNA that is transported into macrophages and might be secreted into blood circulation. Biochem Biophys Res Commun. 2010;398: $723-729$.

21. Guttilla IK, White BA. Coordinate regulation of foxo1 by mir27a, mir-96, and mir-182 in breast cancer cells. J Biol Chem. 2009;284:23204-23216.

22. Ge Q, Brichard S, Yi X, Li QF. MicroRNAs as a new mechanism regulating adipose tissue inflammation in obesity and as a novel therapeutic strategy in the metabolic syndrome. J Immunol Res. 2014;2014:987285.

23. Nieman KM, Kenny HA, Penicka CV, et al. Adipocytes promote ovarian cancer metastasis and provide energy for rapid tumor growth. Nat Med. 2011;17:1498-1503.

24. Salvi A, Abeni E, Portolani N, Barlati S, De Petro G. Human hepatocellular carcinoma cell-specific miRNAs reveal the differential expression of mir-24 and mir-27a in cirrhotic/non-cirrhotic HCC. Int J Oncol. 2013;42:391-402.
25. Liu T, Tang H, Lang YY, Liu M, Li X. MicroRNA-27a functions as an oncogene in gastric adenocarcinoma by targeting prohibitin. Cancer Lett. 2009;273:233-242.

26. Acunzo M, Romano G, Palmieri D, et al. Cross-talk between met and egfr in non-small cell lung cancer involves mir-27a and sprouty2. Proc Natl Acad Sci U S A. 2013;110:8573-8578.

27. Pathi SS, Jutooru I, Chadalapaka G, et al. Gt-094, a no-nsaid, inhibits colon cancer cell growth by activation of a reactive oxygen speciesmicroRNA-27a: Zbtb10-specificity protein pathway. Mol Cancer Res. 2011;9:195-205.

28. Ma YH, Yu SN, Zhao WG, Lu ZH, Chen J. Mir-27a regulates the growth, colony formation and migration of pancreatic cancer cells by targeting sprouty2. Cancer Lett. 2010;298:150-158.

29. Mertens-Talcott SU, Chintharlapalli S, Li X, Safe S. The oncogenic microRNA-27a targets genes that regulate specificity protein transcription factors and the g2-m checkpoint in mda-mb-231 breast cancer cells. Cancer Res. 2007;67:11001-11011.

30. Carter ME, Brunet A. Foxo transcription factors. Curr Biol. 2007;17: R113-R114.

31. Burgering BMT, Medema RH. Decisions on life and death: Foxo forkhead transcription factors are in command when pkb/akt is off duty. J Leukocyte Biol. 2003;73:689-701.

32. Fu Z, Tindall DJ. Foxos, cancer and regulation of apoptosis. Oncogene. 2008;27:2312-2319.

33. Carbajo-Pescador S, Mauriz JL, Garcia-Palomo A, Gonzalez-Gallego J. Foxo proteins: Regulation and molecular targets in liver cancer. Curr Med Chem. 2014;21:1231-1246.

34. Wu K, Ding J, Chen C, et al. Hepatic transforming growth factor beta gives rise to tumor-initiating cells and promotes liver cancer development. Hepatology. 2012;56:2255-2267.

35. Li J, Yang L, Song L, et al. Astrocyte elevated gene-1 is a proliferation promoter in breast cancer via suppressing transcriptional factor foxo1. Oncogene. 2009;28:3188-3196.

36. Kuo YY, Lin HP, Huo C, et al. Caffeic acid phenethyl ester suppresses proliferation and survival of TW2.6 human oral cancer cells via inhibition of Akt signaling. In. J Mol Sci. 2013;14:8801-8817.

37. Modur V, Nagarajan R, Evers BM, Milbrandt J. Foxo proteins regulate tumor necrosis factor-related apoptosis inducing ligand expression - implications for pten mutation in prostate cancer. $J$ Biol Chem. 2002;277:47928-47937.
OncoTargets and Therapy

\section{Publish your work in this journal}

OncoTargets and Therapy is an international, peer-reviewed, open access journal focusing on the pathological basis of all cancers, potential targets for therapy and treatment protocols employed to improve the management of cancer patients. The journal also focuses on the impact of management programs and new therapeutic agents and protocols on

\section{Dovepress}

patient perspectives such as quality of life, adherence and satisfaction The manuscript management system is completely online and includes a very quick and fair peer-review system, which is all easy to use. Visit http://www.dovepress.com/testimonials.php to read real quotes from published authors. 